\title{
Advanced Endoscopic Imaging in Colonic Neoplasia
}

\author{
Timo Rath Nadine Morgenstern Francesco Vitali \\ Raja Atreya Markus F. Neurath \\ Ludwig Demling Endoscopy Center of Excellence, University Hospital Erlangen, \\ Friedrich Alexander University Erlangen-Nuremberg, Erlangen, Germany
}

\section{Keywords}

Endoscopy - Colorectal cancer - Colorectal polyps ·

Endocytoscopy $\cdot$ Endomicroscopy

\begin{abstract}
Background: Endoscopic imaging is a rapidly evolving field with a constant influx of new concepts and technologies. Since the introduction of video endoscopy and subsequently high-definition imaging as the first revolutions in gastrointestinal endoscopy, several technologies of virtual chromoendoscopy have been developed and brought to the market in the past decade, which have shaped and revolutionized for a second time our approach to endoscopic imaging. In parallel to these developments, microscopic imaging technologies, such as endomicroscopy and endocytoscopy, allow us to examine single cells within the mucosa in real time, thereby enabling histological diagnoses during ongoing endoscopy. Summary: In this review, we provide an overview on the technical background of different technologies of advanced endoscopic imaging, and then review and discuss their role and applications for the diagnosis and management of colorectal neoplasms as well as limitations and challenges that exist despite all technological improvements. Key Messages: Technologies of advanced endoscopic imaging have profound impact not only on our imaging capabilities, they are also about to fundamentally change our approach to managing lesions in the gastrointestinal tract: not every lesion found during colonoscopy has to be excised or sent for histopathologic evaluation.
\end{abstract}

However, before this becomes widespread reality, major obstacles such as patient acceptance, adoption by less trained endoscopists, and also legal aspects need to carefully addressed. The development of computer-aided diagnosis and artificial intelligence algorithms hold the potential to overcome the obstacles associated with the concept of optical biopsy and will most likely fundamentally facilitate, shape, and change decision making in the management of colorectal lesions.

(c) 2020 S. Karger AG, Basel

\section{Introduction}

The last decade has brought groundbreaking technological developments into daily endoscopic routine: apart from endoscopes equipped with high-definition (HD) and high-magnification imaging, we now have a variety of digital chromoendoscopy techniques that, by simply pushing a button, enable an enhanced delineation and characterization of neoplastic lesions within the GI tract, microscopic techniques such as confocal laser endomicroscopy and endocytoscopy (EC) allowing for in vivo real-time histology, and artificial intelligence for computer-aided detection and characterization of neoplasms within the GI tract. Before the contribution of the various technologies to better endoscopic visualization of colonic neoplasia is discussed, a brief summary of the technological background of the above-mentioned techniques is presented. 


\section{HD and High-Magnification Endoscopy}

Standard-definition (SD) endoscopes offer a resolution of 100,000-400,000 pixels displayed on a 4:3 monitor (width to height ratio). Advances in the charge-coupled device (CCD) technology and the combination with the metal-oxide semiconductor technology have resulted in smaller chips with an increased number of pixels and increased resolution. By definition, HD endoscopes utilize CCD that exhibit a resolution of 850,000 to 2 million pixels, and the general consensus definition of a HD image is one with more than 650-720 lines of resolution [1] displayed in a 4:3 or 5:4 format. Importantly, in order to ensure HD image quality, every component of the system (e.g., the endoscope video chip, the processor, the monitor, and transmission cables) must be HD compatible [1]. As a drawback of the smaller size of their pixels, HD video chips have a lower light sensitivity compared to SD video chips. Hence, for optimal illumination, the standard light source for HD endoscopy is a $300-\mathrm{W}$ xenon lamp.

Using a built-in movable lens in the tip of the endoscope, high-magnification endoscopes can perform an optical zoom of the mucosa, thereby ensuring that despite magnification of the mucosa (up to 150-fold), the resolution remains stable. This is in contrast to the digital zoom that most of the latest endoscopes offer: here the image on the display is simply enlarged, resulting in decreased pixel density and decreased image quality under digital zoom.

\section{Virtual Chromoendoscopy}

With the development of narrow band imaging (NBI; Olympus, Tokyo, Japan) and its subsequent release to the market in 2005 [2], the concept of virtual chromoendoscopy was introduced and has since greatly shaped the following decade. Virtual chromoendoscopy encompasses 2 different technical approaches: the so-called optical chromoendoscopy is based on the restriction of the light that is emitted into the tissue. For this purpose, an optical filter is used through which only light of dedicated wavelengths pass. Typically, the wavelengths of light that reflect the absorbance maximum of human hemoglobin are chosen, i.e., a shorter blue light with a wavelength of $\lambda=415 \mathrm{~nm}$ and a longer green light with a wave length of $\lambda=540 \mathrm{~nm}$. This restriction to wavelengths that correspond to the absorption maxima of hemoglobin results in an accentuation of the vascular architecture in the picture: the shorter wavelength light accentuates the thin capillary network on the mucosal surface and has brownish appearance while the longer wavelength light has a deeper tissue penetration and highlights thick submucosal blood vessels with a cyan color.

Digital chromoendoscopy, another technique of virtual chromoendoscopy, utilizes digital postprocessing during which the endoscopic image is reconstructed under specific color- and surface-enhancing algorithms, resulting in an improved contrast of the mucosal capillary pattern and enhancement of the mucosal surface pattern morphology [3]. Importantly, both optical and digital chromoendoscopy are so-called "push-of-a-button" technology that allow for virtual staining of the mucosa in real time without any delay. Hence, compared to traditional dye spraying (so-called dye-based chromoendoscopy; DBC), virtual (or dye-less) chromoendoscopy offers the great advantage that mucosal details can be enhanced in real-time by simply pushing a button without the time and cost efforts associated with traditional dye spraying.

To date, virtually all large endoscope manufactures have virtual chromoendoscopy capabilities integrated into their endoscope: NBI (Olympus), i-scan, and i-scan optical enhancement (Pentax, Tokyo, Japan), flexible spectral imaging color enhancement (FICE; Fujifilm, Japan), blue light imaging (BLI) and linked color imaging (LCI;Fujifilm), and SPECTRA (Karl Storz, Tuttlingen, Germany)

\section{Confocal Laser Endomicroscopy}

Confocal laser endomicroscopy (CLE) has been introduced to the market more than 1 decade ago and since then emerged as a cross-sectional imaging technology which enables real-time microscopy of the intestinal mucosa. Technically, CLE is based on the emission of lowpowered blue laser light with a wavelength of $\lambda=415 \mathrm{~nm}$ that is directed through a pinhole onto a defined point of the mucosa [3-5]. The mucosa then produces an autofluorescence signal that is detected by the same device. Importantly, the reflected light again passes through a pinhole thereby ensuring that only light from the plane of interest is detected while virtually no scattered light reaches the detection system. This results in a very high lateral resolution of CLE imaging with an overall magnification of $\times 1,000$ [3-5].

Historically, 2 different CE-marked CLE devices were available on the market: one CLE device is based on the utilization of probes (probe-based CLE, pCLE; Mauna Kea, Paris, France) consisting of thousands of optical fibers that allow to assess virtually any mucosal surface within the human body. The probes can be advanced through the working channel of any standard endoscope, and different probes are available for various indications: probes for the upper GI tract (esophagus, stomach, and duodenum), lower GI tract (rectum, colon, and terminal ileum), biliary tract, pancreas (needlebased CLE), and urinary and respiratory tract. Technically, pCLE utilizes a fixed laser power with a confocal imaging depth in the lower GI tract of 55-65 $\mu \mathrm{m}$ and a resolution of $1 \mu \mathrm{m}$. The field of view encompasses 
$240 \mu \mathrm{m}$ with an image acquisition rate of 8 frames per second, thereby enabling real-time microscopic video imaging of the mucosa [3-5]. As a major advantage of this system, pCLE imaging can be readily incorporated into an existing endoscopy setup, and extensive experience is available with pCLE imaging.

In the past, a CLE system integrated into the tip of a high-resolution endoscope was available (endoscopebased CLE, eCLE; Pentax Medical, Tokyo, Japan), and although still used in many CLE trials, this system is no longer commercially available. Technically, laser power can be manually adjusted with eCLE, and, consequently, imaging depth can also be dynamically adjusted ranging from 0 to $250 \mu \mathrm{m}$. The eCLE system collects images at a manually adjustable scan rate of either 1.6 frames per second with a resolution of $1,024 \times 512$ pixels or at 0.8 frames per second with a resolution of $1,024 \times 1,024$ pixels. Lateral resolution of eCLE is $0.7 \mu \mathrm{m}$ with a field of view of $475 \times 475 \mu \mathrm{m}$ [3-5]. Given the rather low image acquisition rate, eCLE imaging is prone to motion artifacts. On the other hand, using eCLE, the working channel can still be used, thereby rendering this system ideal for direct comparison of CLE-based "optical" biopsy and conventional "physical" biopsy.

\section{Endocytoscopy}

EC is based on the principle of contact light microscopy and typically uses a continuous zoom mechanism similar to magnification endoscopy [6-8]. Prior to EC imaging, cells are typically stained with a topically applied dye or a dye mixture [6-8]. After staining of the mucosa, the objective lens is brought into contact with the mucosa followed by light emission into the cells which then return a scattered light, thereby enabling cytological observation of the superficial mucosal layer. To date, different systems have been used in the various studies in the field. Similar to CLE, integration of the endoscopy function into the tip of an endoscope has been realized most recently and represents the most commonly used application of endocytoscopic imaging [7-9]. By integration of the EC function into a HD high-resolution gastroscope and colonoscope (GIF H-290 EC and GIF H-290 ECI; both Olympus), it is possible to dynamically visualize and monitor single cells within the upper and lower GI tract with $\times 520$ magnification in real time by simply controlling EC function with the hand lever of the endoscope. Importantly, imaging depth can be manually and continuously adjusted from normal overview to magnification endoscopy to EC. This enables instantaneous monitoring of single cells at defined points of interest without the necessity to change the equipment. Both, the gastroscope (GIF H-290 EC) and the colonoscope (GIF H-290 ECI) are otherwise fully equipped endoscopes with a field of view of $140^{\circ}$ and a working channel of 2.2 and $3.2 \mathrm{~mm}$, respectively, thereby enabling also the use as endoscopes for clinical routine. Importantly, both endoscopes are also equipped with NBI and this, in combination with the EC function, represents a powerful tool to assess the vasculature at single-cell level.

Apart from this integrated endocytoscope function, probe-based EC has been developed and used in the past $[7,10]$. So far, 2 different EC probes have been described, providing either 450 -fold (XEC 300F) or 1,390-fold (XEC $120 \mathrm{U})$ magnification with a horizontal observation field of $300 \times 300 \mu \mathrm{m}\left(0.09 \mathrm{~mm}^{2}\right)$ for the 450 -fold magnification probe and of $120 \times 120 \mu \mathrm{m}$ for the 1,390-fold magnification probe [3]. The probes are $3.2 \mathrm{~mm}$ in diameter and thereby can be used with most standard endoscopes. Similar to what is already discussed with different CLE devices, one of the major advantages of the probe-based EC system is the fact that it can be readily incorporated into an existing endoscopy setup whereas the integrated EC devices allow to simultaneously take biopsies and thus to directly compare EC imaging with histopathological results while they can be used as standard endoscopes at the same time.

\section{Role and Applications of Advanced Endoscopic Imaging in Colorectal Neoplasms}

\section{HD and High-Magnification Endoscopy}

In the past, various studies have assessed whether HD endoscopy can lead to an improved visualization and detection of colorectal neoplasms and especially colorectal polyps. These studies have been summarized in a metaanalysis including over 4,000 patients comparing the diagnostic yield in terms of detection of colorectal polyps between SD and HD [11]. As shown herein, HD exhibited only a marginally increased yield for the detection of colorectal polyps of $3.5 \%$ compared to SD, and the number needed to treat was calculated to be 28 [11]. However, while HD offers only an incremental increase in the detection of colorectal polyps as precursors of sporadic colorectal cancer, the effect of HD imaging on the detection of colitis-associated dysplasia is considerable. Typically, and unlike sporadic colorectal carcinogenesis, dysplasia in patients with inflammatory bowel disease develops as flat lesions surrounded by inflamed mucosa instead of lesions protruding into the intestinal lumen. As shown in a retrospective cohort study, HD endoscopy was able to detect significantly more neoplastic lesions than SD endoscopy with an adjusted prevalence ratio of detecting dysplastic lesions on targeted biopsies of 2.99 for HD colonoscopy [12].

While HD imaging, therefore, increases the detection of colonic neoplasms especially in patients with inflammatory bowel disease, the predominant role of optical 
magnification certainly lies in the characterization of neoplastic lesions: as shown in one of the earliest studies by Kudo et al. [13], analysis of the pit pattern under optical magnification allowed to distinguish neoplastic from nonneoplastic colorectal pathology. This study was clearly one of the first studies that founded and stimulated the field of "optical biopsy" or "real-time histology" with the overall aim to predict histology with technologies of advanced imaging instantaneously during ongoing endoscopy.

The relevance of optical magnification endoscopy is not limited to the prediction of histology of sporadic colorectal neoplasia, it has also been extended to and confirmed in colitis-associated dysplasia. In one of the earliest studies in this field, Kiesslich et al. [14] directly compared DBC combined with optical magnification to SD endoscopy for the detection of colorectal neoplasia in a large cohort of patients with ulcerative colitis. As shown in this landmark study, DBC with magnification endoscopy significantly improved the early detection of intraepithelial neoplasia and colitis-associated cancer as compared to SD endoscopy [14]. Subsequently, these findings have been corroborated by several other studies in the field. As shown in a prospective controlled trial including 700 patients with chronic ulcerative colitis, magnification together with DBC led to significantly higher detection rates of intraepithelial neoplasia compared to SD colonoscopy: out of 67 lesions with intraepithelial neoplasia, 53 lesions were only found with magnification endoscopy combined with DBC [15]. Overall, these studies support the concept that especially in colitis-associated dysplasia, HD endoscopy and optical magnification endoscopy can lead to improved demarcation of a colorectal neoplasm from the surrounding inflamed mucosa with superior detection rates of colitis-associated neoplasia as compared to conventional SD white light endoscopy, and improved visualization of the mucosal pit pattern and vascular pattern under these techniques is certainly among the key components for the enhanced detection.

\section{Virtual Chromoendoscopy}

NBI was the first commercially available narrow spectrum technology, and, hence, most of the studies on applications of virtual chromoendoscopy for assessing colorectal neoplasms are based on NBI. In the following, we review the role of virtual chromoendoscopy for both: polyp detection and characterization.

\section{Polyp Detection}

Through enhancement of the vascular and surface pattern, NBI (and other virtual chromoendoscopy techniques) can potentially lead to a better demarcation of lesions thereby facilitating detection. However, various studies have shown that NBI does not improve the detection of colorectal polyps when compared to HD colonoscopy, and these results have been confirmed and corroborated in several meta-analyses [16-20].

It is important to keep in mind that these studies were, at least in part, performed with first-generation NBI systems (Lucera Spectrum and Exera II series), which exhibited an NBI image that was considerably darker than the white light image. Recent studies using the latest NBI generations (Lucera Elite and Exera III) with technical improvement in the light source and thus brighter images compared to first-generation NBI do indeed show that "bright" NBI can lead to enhanced detection of colorectal polyps [21-23]. Clearly, further studies and meta-analyses are needed before final conclusions on the role of NBI for polyp detection can be drawn.

Another virtual chromoendoscopy, i-scan imaging, has potential to improve the adenoma detection rate. Indeed, several lines of evidence support the concept of an increased detection of colorectal lesions under i-scan imaging compared to HD colonoscopy:

In a large prospective study by Bowman et al. [24] on 1,936 patients, significantly more adenomas, and, importantly, also significantly more advanced adenomas, were found using i-scan imaging than HD white-light endoscopy. Another prospective randomized controlled trial used a tandem colonoscopy approach to show that i-scan tone enhancement can significantly increase adenoma detection and also identifies significantly more missed adenomas per subject than the HD white light approach [25]. Apart from that, various studies show that i-scan imaging can significantly increase the detection not only of colorectal polyps [26,27], but also of cancerous lesions when compared to SD colonoscopy [28].

As one of the most recently introduced technologies, LCI (Fujifilm) increases color contrast by utilizing shortwavelength narrow-band laser light combined with white laser light on the basis of BLI technology. However, in contrast to NBI and BLI, the luminal brightness of LCI is not reduced compared to the white-light image, and, hence, LCI is another promising technique to improve the visibility of colonic neoplasms. Indeed, as recently shown in the first well-designed trials, LCI can reduce the rate of missed adenomas [29] (PMID: 29539651) and significantly increase adenoma detection [30], even of lesions that can be challenging to detect, such as sessile serrated adenomas (SSA) [30, 31] or flat lesions [32], or lesions at difficult and critical locations such as the right colon [29]. Hence, LCI is another virtual chromoendoscopy technique that holds the potential to enhance the detection of clinically relevant lesions and therefore might contribute to increase the effectiveness and quality of screening and surveillance colonoscopy. 


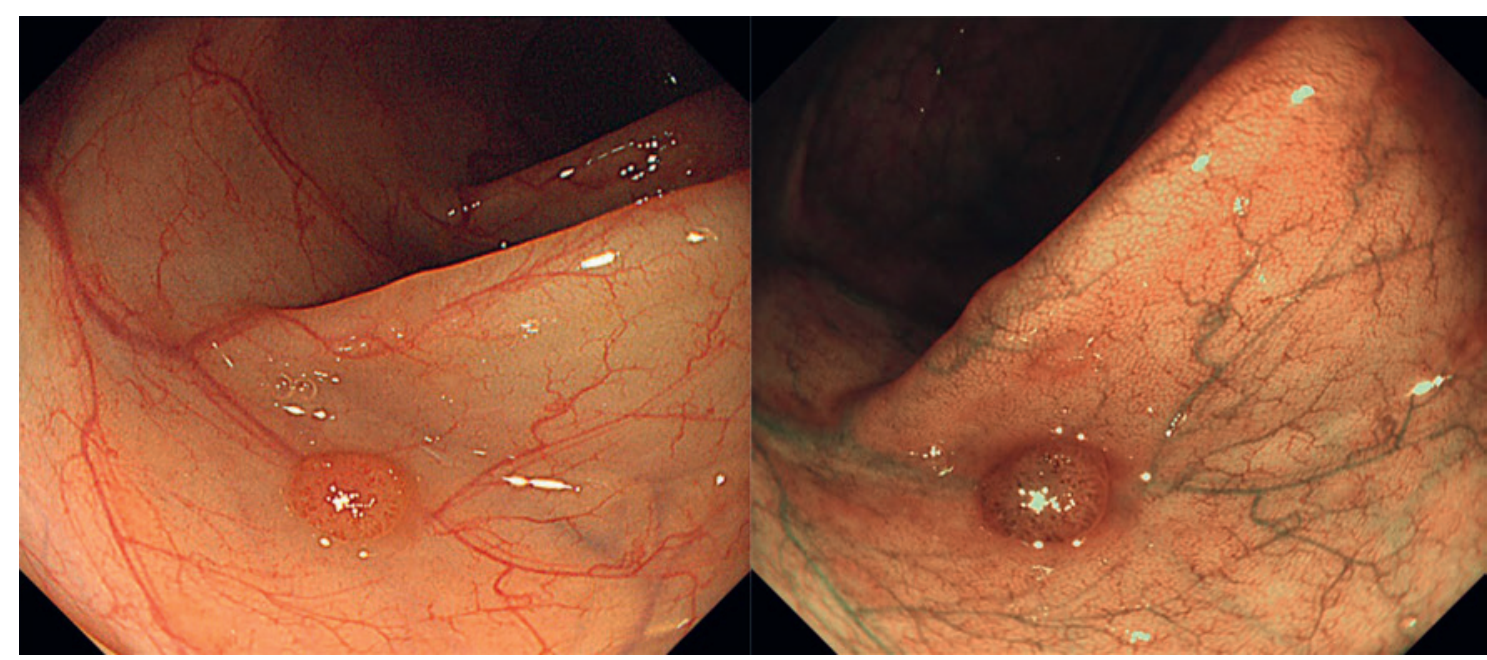

Fig. 1. Virtual chromoendoscopy with narrow band imaging (NBI). NBI is an optical filter technology in which wavelengths of light that reflect the absorbance maximum of human hemoglobin are emitted into the tissue, i.e., a shorter blue light with a wavelength of $\lambda=415 \mathrm{~nm}$ and a longer green light with a wavelength of $\lambda=540 \mathrm{~nm}$. This results in an accentuation of the vascular architecture (right picture) of colorectal polyps compared to HD white light imaging (left picture).

\section{Polyp Characterization}

Apart from polyp detection, accurate in vivo polyp characterization and prediction of its histology is equally important. One could argue that every polyp found during colonoscopy will be removed anyway with a limited clinical consequence of the in vivo prediction of its histology by advanced imaging technologies. However, at the same time, a cross-sectional analysis of a large colonoscopy database for polyp histology calculated that endoscopic diagnosis of the histology of diminutive polyps during colonoscopy instead of removing the lesions with subsequent pathologic examination would result in substantial up-front cost savings exceeding more than 1 billion per year alone in the US [33].

Further, diminutive polyps (polyps $\leq 5 \mathrm{~mm}$ ) have a very high prevalence and can be found in $>50 \%$ of the population screened $[34,35]$ while, at the same time, the prevalence of advanced histological features and cancer within diminutive polyps is very low $[36,37]$, and their cancer prevalence ranges between 0 and $0.08 \%[38,39]$.

Based on these considerations, the American Society for Gastrointestinal Endoscopy (ASGE) proposed 2 new strategies for the management of diminutive polyps in a PIVI (Preservation and Incorporation of Valuable Endoscopic Innovations) [40]: in the so-called resect-anddiscard strategy, adenomatous diminutive polyps would be endoscopically removed but not sent for histopathology, thereby reducing the costs associated with histopathological assessment [40]. In the leave-in-place strategy, suspected hyperplastic diminutive polyps would not be removed but left in situ instead (since they are nonadenomatous), thereby reducing the costs associated with removal and histopathologic assessment [40]. A prerequisite for both of these studies is a technique that enables an accurate in vivo prediction of polyp histology. Using Markov modeling to compare the cost-effectiveness of universal pathology evaluations with a resect and discard policy, it was shown that these strategies resulted in a substantial economic benefit without an impact on efficacy: using the resect and discard policy for diminutive colorectal polyps would result in cost savings of USD 25/person without affected screening efficacy [41]. Projected onto the US population, this approach would result in undiscounted annual savings of USD 33 million [41].

Hence, in the past decade, numerous studies on the different technologies of virtual chromoendoscopy have been conducted analyzing whether the various techniques can be used to predict polyp histology in vivo during ongoing endoscopy according to the criteria as set forth by the ASGE PIVI statement.

Since NBI was the first virtual chromoendoscopy technique introduced in the market, most of the studies on optical diagnosis of colorectal polyps have been performed with NBI. An example of polyp appearance under $\mathrm{HD}$ white light and NBI can be found in Figure 1. For the differentiation of polyps based on their endoscopic appearance, the majority of studies used the so-called NICE (NBI International Colorectal Endoscopic) classification (validation of a simple classification system for endoscopic diagnosis of small colorectal polyps using NBI). The NICE classification facilitates the differentiation between hyperplastic polyps (HP) and adenomatous polyps and colorectal cancer based on the surface and vascular pat- 


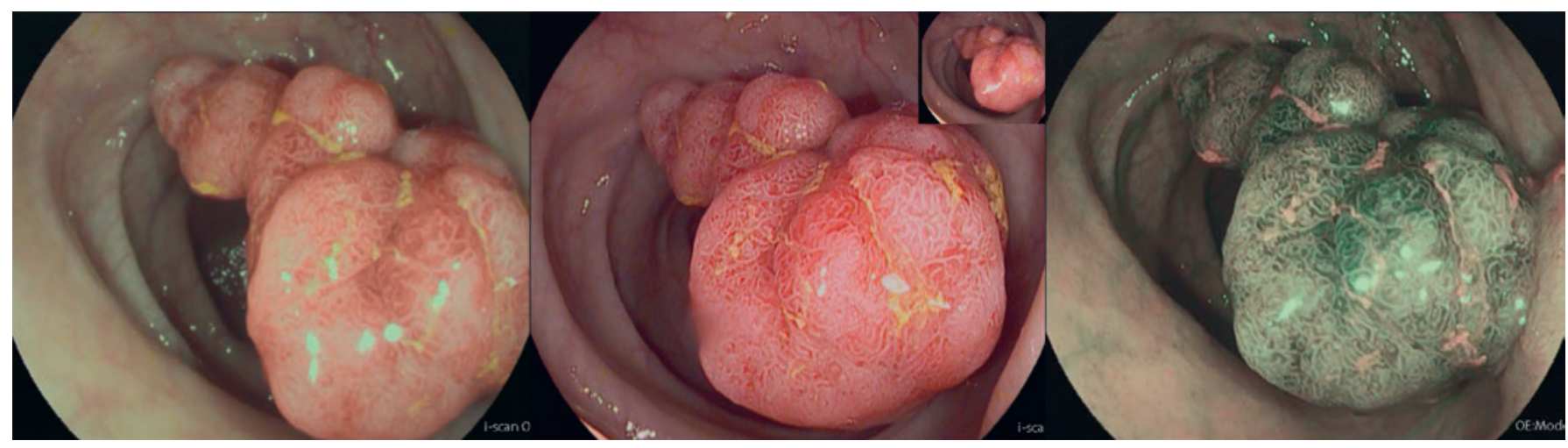

Fig. 2. Virtual chromoendoscopy with i-scan. By improving dark light contrast, i-scan imaging can enhance the surface structure of the mucosa leading to an overall increase in sharpness and clarity of the mucosal pit pattern (i-scan 1 or surface enhancement, middle picture) compared to HD white light images (left picture). i-scan optical enhancement (right picture) is an optical filter technology in which wavelength-restricted light corresponding to the absorption maxima of hemoglobin is emitted into the mucosa, thereby enhancing the contrast of blood vessels for improved visualization of the vascular pattern.

tern and the color of the lesions under NBI [42]. As shown in a recent meta-analysis including 19 studies on NBI collectively evaluating 4,013 diminutive colorectal polyps, the negative predictive value (NPV) for ruling out adenomatous histology under NBI was $91 \%$, thereby exceeding the cutoff of $90 \%$ as set forth by the ASGE for adopting the leave-in-place strategy [43]. Further, 10 studies including 3,082 patients reported on the degree of agreement with histopathology when assigning postpolypectomy surveillance intervals based on combining real-time NBI optical biopsy of colorectal polyps $\leq 5 \mathrm{~mm}$ with histopathologic assessment of polyps $>5 \mathrm{~mm}$ using the US Multi-Society Task Force (MSTF) postpolypectomy surveillance intervals [44] and found a pooled agreement in the assignment of postpolypectomy surveillance intervals between histopathology and optical biopsy of $89 \%$, thereby just barely missing the cutoff set forth by the ASGE $[40,43]$.

For i-scan, fewer data are available. At the time of the systemic review and meta-analysis by the ASGE technological committee in 2015, 8 i-scan studies reported or provided information on the negative prediction of optical biopsies performed using i-scan for predicting adenomatous polyp histology of small and diminutive colorectal polyp evaluation (almost 1,000 patients in total). Collectively, these studies show that optical biopsy with i-scan exceeds the threshold for ruling out adenomatous histology with an NPV of $96 \%$ from experienced endoscopists. However, as shown at the same time, this threshold cannot be met by inexperienced endoscopists, thereby illustrating that some level of training is required when performing optical biopsy. However, since the publication of this meta-analysis in 2015, several other studies assessed the prediction of histology of diminutive colorectal polyps by i-scan imaging [45-47] and confirmed that i-scan imaging can rule out adenomatous histology with very high NPV (>90\%) and can also accurately predict postpolypectomy surveillance intervals [45-47]. Importantly, as recently shown, i-scan optical enhancement, a novel preprocessing optical chromoendoscopy technology in which optical filters reduce the emitted light to wavelengths that correspond to the absorption maximum of hemoglobin, can accurately predict polyp histology with an NPV $>90 \%$ when using the above-mentioned NICE criteria and also has a high concordance with histopathology for the prediction of postpolypectomy surveillance intervals [46]. Further, as shown in this report, junior endoscopists achieved a comparable diagnostic performance for predicting polyp histology with i-scan optical enhancement compared to expert endoscopists, thereby suggesting that polyp differentiation with i-scan optical enhancement requires only a short learning curve [46]. An example of the appearance of large colorectal polyps under HD white-light and i-scan imaging can be found in Figure 2.

Eight studies reported diagnostic performances according to the ASGE PIVI thresholds for optical biopsy performed by FICE for differentiation of HP versus adenomatous diminutive polyps, collectively evaluating 1,243 lesions. The pooled NPV for ruling out adenomatous histology with FICE was $80 \%$, with a significant degree of heterogeneity across studies. However, when performing subgroup analysis based on the experience of the various endoscopists, diagnostic performance was not improved. Nevertheless, the use of magnification did improve FICE performance with an NPV of $85 \%$. Only 2 studies assessed the concordance in assigning postpolypectomy surveillance intervals and reported a degree of agreement of FICE optical biopsy with histopathology of 100 and $97 \%[48,49]$. 


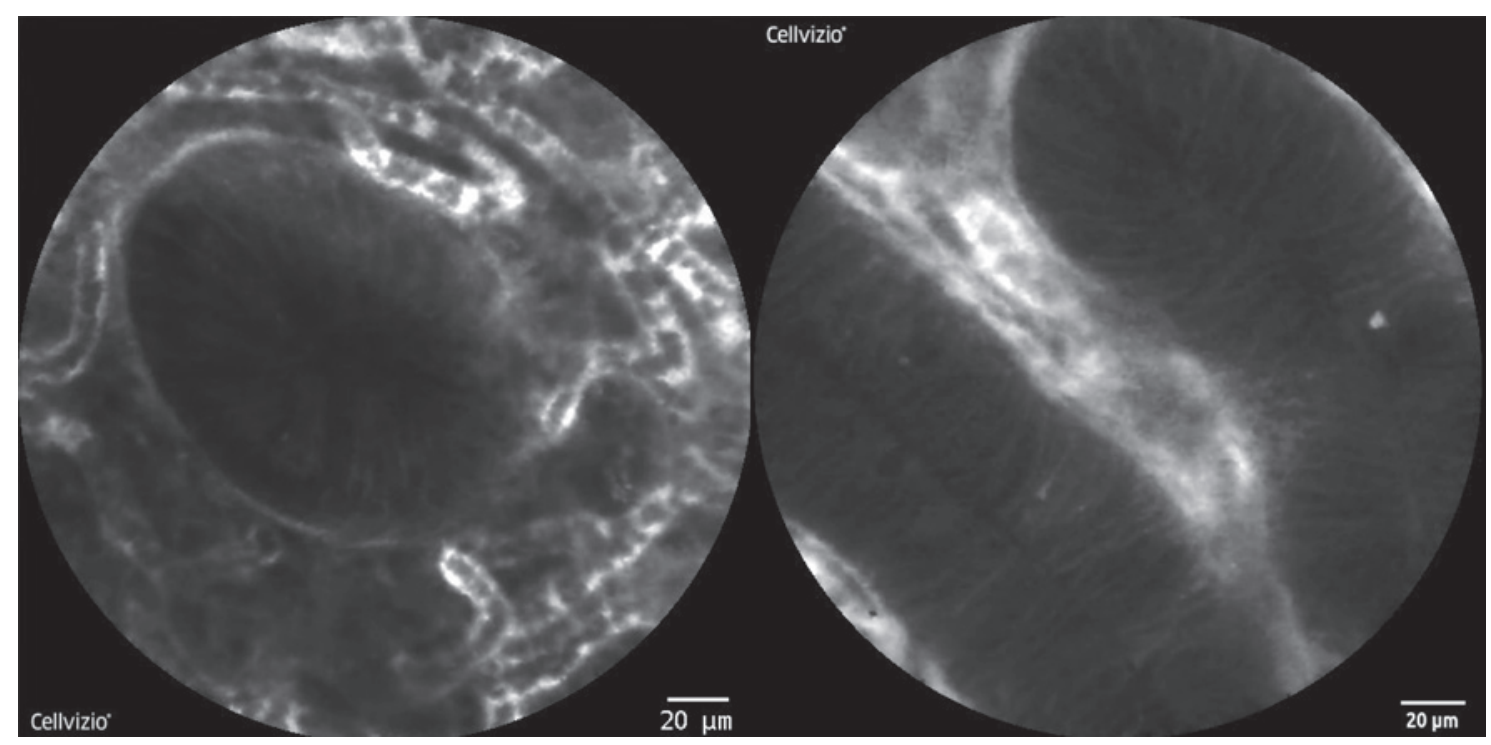

Fig. 3. Confocal laser endomicroscopy (CLE) allows visualizing the mucosa with 1,000-fold magnification. Normal colonic crypts are round with a small and dark central lumen surrounded by fluorescein-containing vessels (left picture). In CLE, colonic adenomas are characterized by tubular-shaped and elongated crypts with loss of goblet cells and a thin and irregular lamina propria.

Although these results are certainly encouraging regarding the accuracy of optical diagnosis and prediction of polyp histology by advanced imaging technologies, several aspects require special attention: the first one refers to the fact that the vast majority of studies were performed by experienced endoscopists with a certain expertise in advanced imaging at academic centers. Looking then at data from studies that included community-derived endoscopists, real-time optical biopsy does not seem to meet the above-discussed thresholds yet [5053]. Secondly, patient support and acceptance of optical diagnosis appears to be limited so far, and, also, only about $2 / 3$ of gastroenterologists would be willing to adopt optical diagnosis and the associated ASGE strategies for handling diminutive polyps in their own practice $[54,55]$, indicating that especially medicolegal concerns and lack of financial incentives are key aspects to clarify before these strategies can become widely available. Thirdly, the vast majority of studies on virtual chromoendoscopy have focused on the dichotomous distinction between adenomatous polyps and HP. With the continuously growing awareness of the neoplastic potential of SSA and polyps (SSA/P), and their still insufficient detection especially by low-detecting or inexperienced endoscopists [56-59], virtual chromoendoscopy still has to prove that it leads to a better demarcation, detection, and characterization of these challenging lesions. First results are quite sobering. As shown in a recent study from an academic center with expertise in optical diagnosis, accurate optical diagnosis of small SSA/P was possible in only $24.4 \%$, and although diag- nostic accuracy improved over time, this was still insufficient for correct optical diagnosis over a 4-year observation period [60].

\section{Confocal Laser Endomicroscopy}

Due to the fact that CLE has a rather limited field of view but allows for cross-sectional high-resolution imaging at microscopic level with 1,000-fold magnification, this technique is not primarily used for the detection of colorectal neoplasms but enables microscopic characterization and differentiation of neoplastic lesions. Soon after the introduction of CLE into the market, Kiesslich et al. [61] developed criteria for the differentiation of normal mucosa versus regenerative mucosa from neoplasia based on their crypt and vascular pattern on eCLE. Importantly, in this seminal pilot study, $>13,000$ confocal images were analyzed, and it was shown that CLE was able to predict neoplastic changes with an overall accuracy $>99 \%$. In the following, key features of the appearance of colorectal polyps under CLE imaging have been further defined and characterized [61, 62].

As such, neoplastic mucosa exhibits the following characteristics on CLE imaging: tubular-shaped and elongated crypts with a loss of goblet cells, a thin and irregular lamina propria, and a generally darkened image which is most likely the result of the volume loss of the lamina propria with reduced vascularization $[61,63]$. Figure 3 illustrates the appearance of healthy mucosa and a tubular adenoma on pCLE. Subsequently, studies were able to corroborate this high accuracy of CLE imaging for the characterization and prediction of polyp histology. 
Buchner et al. [63] directly compared the diagnostic performance of pCLE and virtual chromoendoscopy (NBI and FICE) in predicting the histology of 119 polyps in total. As shown in this report, optical diagnosis with pCLE had a significantly higher sensitivity than virtual chromoendoscopy (91 vs. $71 \%$, respectively), while specificity was unchanged between CLE and virtual chromoendoscopy. Although several studies have performed offline postprocedural evaluation of CLE images [64-67], relatively few studies have assessed whether CLE can differentiate colorectal polyps in real time according to the ASGE PIVI thresholds $[61,62,68,69]$. Importantly, these studies exhibited considerable variability in terms of the CLE device utilized (pCLE vs. eCLE), the contrast agent used (systemic administration of fluorescein as a nonnuclear dye versus acriflavine as a dye-staining agent), and the overall study setup (inclusion of normal mucosa vs. analysis of polyps only). In line with this heterogeneity of the study setup, results have also shown considerable NPV variation for adenomatous histology ranging from 79 to $100 \%$.

Apart from the binary distinction between neoplastic and nonneoplastic polyps, other studies assessed the feasibility of pCLE imaging for evaluating submucosal invasion of colorectal cancer. As such, Kim et al. [70] just recently analyzed 51 lesions from 31 patients and found that in those lesions with submucosal carcinoma infiltration, dark and irregular cell nests with irregular cell architecture and little or no mucin were visible on pCLE. Based on correlations with pathological findings, sensitivity, specificity, and accuracy of the classification of submucosal carcinoma infiltration by 2 observers were 91.7, 86.8, and $88.0 \%$, respectively, in this study [70].

Although this study clearly evokes the possibility that pCLE assessment of colorectal polyps and lesions with suspected submucosal invasion can directly influence clinical and therapeutic decision making, e.g., endoscopic mucosal resection versus endoscopic submucosal dissection versus surgery, larger multicentric studies are clearly needed before far-reaching conclusions can be drawn from these findings.

\section{Endocytoscopy}

With the possibility to continuously zoom from standard overview to 520 -fold magnification and to visualize the vascular and mucosal pit pattern in great detail, first studies assessed whether EC can differentiate between nonneoplastic, neoplastic, and cancerous tissue. In one of the first studies, Kudo et al. [71] developed a novel EC classification system with the following categories: EC 1a: normal mucosa; EC 1b: hyperplastic mucosa; EC 2: dysplasia; and EC $3 \mathrm{~b}$ : massive invasive submucosal cancer. Using this classification in a total of 213 lesions from 196 patients, the authors found that EC was able to differenti- ate nonneoplastic from neoplastic lesions with a sensitivity of $100 \%$ and a specificity of $100 \%$, and lesions with massive submucosal invasion from other neoplastic lesions with a sensitivity of $90.1 \%$ and a specificity of $99.2 \%$ [71].

Subsequently, the same group extended these observations and evaluated 198 colorectal lesions based on the morphology of their vascular EC pattern [72]. For this purpose, EC appearance of the microvasculature was categorized as follows: EC-V1: obscure surface microvessels; EC-V2: clear surface microvessels of a uniform caliber and arrangement; and EC-V3: dilated surface microvessels of a nonhomogeneous caliber or arrangement [72]. As shown in this report, the EC-V1 vascular pattern exhibited a sensitivity, specificity, and accuracy for diagnosing HP of 95.5, 99.4, and 99.0\%, respectively, and although the diagnostic performance was slightly impaired in predicting invasive cancer, the diagnostic accuracy of the EC-V pattern for predicting invasive cancer was comparable to other imaging modalities [72].

In the following, the same group of authors then focused on the prediction of submucosal invasion of large polyps $(\geq 10 \mathrm{~mm})$ and therefore evaluated lesions using EC based on their pit structure concomitant with their nuclear morphology [73]. As shown in this report, EC resulted in an excellent diagnostic accuracy for predicting submucosal invasion, especially regarding laterally spreading nongranular tumors, and, importantly, diagnostic accuracy for these lesions was significantly higher using the EC classification than the pit pattern classification [73]. With NBI now integrated into the latest EC generation (GIF H-290 EC and GIF H-290 ECI; both Olympus), it can be expected that the precise endoscopic characterization of premalignant and malignant lesions will be further facilitated. Figure 4 shows EC images with NBI and topical dye spraying.

As discussed above, the detection as well as in the precise and accurate endoscopic characterization of serrated lesions currently represent a challenge. In the recent past, emerging evidence suggested that EC holds the potential to close this gap by accurately predicting serrated histology. In one of the earliest studies in the field, EC was used to assess the crypt lumen and nuclear morphology to differentiate serrated lesions [74]. As shown in this report, the presence of star-like lumens was a significant characteristic of HP while oval lumina were significant characteristics of SSA/P. In contrast, the lumina of traditional serrated adenomas were serrated or villous, with both shapes seen only in traditional serrated adenomas, and features significantly differentiating traditional serrated adenomas from HP and SSA/P were the presence of fusiform nuclei and villous and serrated lumina [74].

These findings have been corroborated by other studies using a similar approach. As shown recently, EC as- 


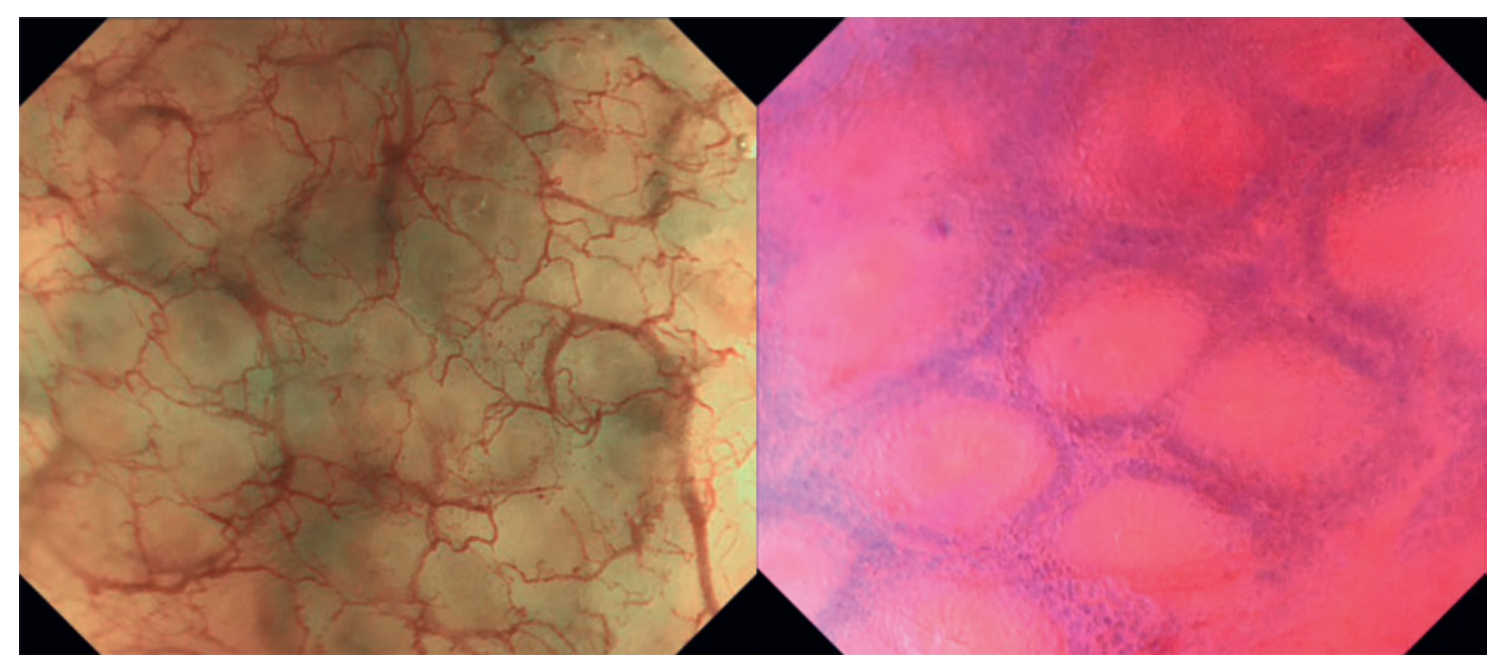

Fig. 4. Endocytoscopy (EC) enables 520-fold magnification of the mucosa and is based on the principle of contact microscopy. Left picture: narrow band imaging during EC enables visualization of the colonic microvasculature. Right picture: EC of healthy colonic mucosa after topical staining with contrast agent shows regular-shaped crypts without significant cell infiltrates in the lamina propria.

sessment and measurement of the crypt lumen helped to differentiate serrated lesions from HP and from normal mucosa [75]. As such, a stepwise increase in the mean luminal diameter from normal mucosa over HP to SSA/P was observed under EC imaging [75]. Using specific cutoffs of the mean luminal diameter, EC enabled differentiation of SSA/P from HP with a sensitivity and specificity of 80 and $77.3 \%$, respectively, and of HP from normal mucosa with a sensitivity and specificity of 98.5 and $100 \%$, respectively [75].

Taken together, these results culminated in the development of computer-aided diagnosis algorithms allowing for automated histological characterization of colorectal lesions in real time by EC and artificial intelligence. First reports have successfully shown that automated differentiation of colorectal polyps using EC combined with artificial intelligence provides a fully automated prompt classification of colorectal polyps with excellent sensitivity, accuracy, and objectivity [76-79]. Based on these results, it can be expected that the combination of EC with computer-aided diagnosis turns into a powerful tool facilitating decision making during routine colonoscopy in the near future.

\section{Conclusion}

During the past decade, various new technologies have been introduced into the market which facilitate the detection and characterization of colorectal lesions. To date, studies clearly show that in expert hands, virtual chromoendoscopy allows to accurately predict pol- yp histology. This concept of optical biopsy holds the potential to fundamentally change our approach to manage especially small and diminutive lesions in the gastrointestinal tract in that way that not every lesion found during colonoscopy will be removed or sent for histopathological evaluation. However, before this becomes widespread reality, major obstacles such as patient acceptance, adoption by less-trained endoscopists, as well as legal aspects need to be addressed carefully. In parallel, microscopic techniques have been introduced in clinical practice, and especially with the combination of virtual chromoendoscopy with microscopic imaging, which was realized in the latest generation of endocytoscopes, the field of optical biopsy is attracting further attention. Therefore, endoscopy is about to close the gap to pathology and enables true "endopathology." Finally, the development of computer-aided diagnosis and artificial intelligence algorithms may potentially overcome the major obstacles associated with the concept of optical biopsy and will most likely fundamentally shape and change decision making in the management of colorectal lesions.

Altogether, these technologies have profound impact not only on our imaging capabilities, they also pave the way for fundamental changes in our approach to lesion management in the gastrointestinal tract. In this review, we provide an overview of the technical background of different technologies of advanced endoscopic imaging and then review and discuss their role and applications for the diagnosis and management of colorectal neoplasms as well as limitations and challenges that exist despite all technological improvements. 


\section{Statement of Ethics}

Since this is a review article, institutional approval and patient consent were not required.

\section{Disclosure Statement}

T.R. has received equipment loans from and serves as a consultant for Pentax Medical, Olympus Medical, and Mauna Kea Technologies. All other authors have no conflict of interest to disclose.

\section{Funding Sources}

The authors received no funding for this paper.

\section{Author Contributions}

T.R. wrote the manuscript. F.V., N.M., R.A., and M.F.N. critically revised the manuscript.

\section{References}

1 Bhat YM, Abu Dayyeh BK, Chauhan SS, Gottlieb KT, Hwang JH, Komanduri S, et al.; ASGE Technology Committee. High-definition and high-magnification endoscopes. Gastrointest Endosc. 2014 Dec;80(6):919-27.

2 Gono K. Narrow Band Imaging: Technology Basis and Research and Development History. Clin Endosc. 2015 Nov;48(6):476-80.

3 Rath T, Tontini GE, Neurath MF, Neumann H. From the surface to the single cell: novel endoscopic approaches in inflammatory bowel disease. World J Gastroenterol. 2015 Oct;21(40):11260-72.

4 Klenske E, Neurath MF, Atreya R, Rath T. Molecular imaging in gastroenterology: A route for personalized endoscopy. Dig Dis Liver. 2018 Sep;50(9):878-85.

5 Rath T, Kiesslich R, Neurath MF, Atreya R. Molecular imaging within the lower gastrointestinal tract: From feasibility to future. Dig Endosc. 2018 Nov;30(6):730-8.

6 Goetz M, Malek NP, Kiesslich R. Microscopic imaging in endoscopy: endomicroscopy and endocytoscopy. Nat Rev Gastroenterol Hepatol. 2014 Jan;11(1):11-8.

7 Inoue H, Kudo SE, Shiokawa A. Technology insight: laser-scanning confocal microscopy and endocytoscopy for cellular observation of the gastrointestinal tract. Nat Clin Pract Gastroenterol Hepatol. 2005 Jan;2(1):31-7.

8 Inoue $\mathrm{H}$, Yokoyama A, Kudo SE. [Ultrahigh magnifying endoscopy: development of CM double staining for endocytoscopy and its safety]. Nihon Rinsho. 2010 Jul;68(7):124752. Japanese.

9 Sasajima K, Kudo SE, Inoue H, Takeuchi T, Kashida H, Hidaka E, et al. Real-time in vivo virtual histology of colorectal lesions when using the endocytoscopy system. Gastrointest Endosc. 2006 Jun;63(7):1010-7.

10 Eberl T, Jechart G, Probst A, Golczyk M, Bittinger M, Scheubel R, et al. Can an endocytoscope system (ECS) predict histology in neoplastic lesions? Endoscopy. 2007 Jun;39(6): 497-501.

11 Subramanian V, Mannath J, Hawkey CJ, Ragunath K. High definition colonoscopy vs. standard video endoscopy for the detection of colonic polyps: a meta-analysis. Endoscopy. 2011 Jun;43(6):499-505.

12 Subramanian V, Ramappa V, Telakis E, Mannath J, Jawhari AU, Hawkey CJ, et al. Comparison of high definition with standard white light endoscopy for detection of dysplastic lesions during surveillance colonoscopy in patients with colonic inflammatory bowel disease. Inflamm Bowel Dis. 2013 Feb;19(2):350-5.

13 Kudo S, Tamura S, Nakajima T, Yamano H, Kusaka H, Watanabe H. Diagnosis of colorectal tumorous lesions by magnifying endoscopy. Gastrointest Endosc. 1996 Jul;44(1):8-14.

14 Kiesslich R, Fritsch J, Holtmann M, Koehler $\mathrm{HH}$, Stolte M, Kanzler S, et al. Methylene blue-aided chromoendoscopy for the detection of intraepithelial neoplasia and colon cancer in ulcerative colitis. Gastroenterology. 2003 Apr; 124(4):880-8.

15 Hurlstone DP, Sanders DS, Lobo AJ, McAlindon ME, Cross SS. Indigo carmine-assisted high-magnification chromoscopic colonoscopy for the detection and characterisation of intraepithelial neoplasia in ulcerative colitis: a prospective evaluation. Endoscopy. $2005 \mathrm{Dec}$; 37(12):1186-92.

16 Dinesen L, Chua TJ, Kaffes AJ. Meta-analysis of narrow-band imaging versus conventional colonoscopy for adenoma detection. Gastrointest Endosc. 2012 Mar;75(3):604-11.

17 Jin XF, Chai TH, Shi JW, Yang XC, Sun QY. Meta-analysis for evaluating the accuracy of endoscopy with narrow band imaging in detecting colorectal adenomas. J Gastroenterol Hepatol. 2012 May;27(5):882-7.

18 Nagorni A, Bjelakovic G, Petrovic B. Narrow band imaging versus conventional white light colonoscopy for the detection of colorectal polyps. Cochrane Database Syst Rev. 2012 Jan;1:CD008361.

19 Omata F, Ohde S, Deshpande GA, Kobayashi D, Masuda K, Fukui T. Image-enhanced, chromo, and cap-assisted colonoscopy for improving adenoma/neoplasia detection rate: a systematic review and meta-analysis. Scand J Gastroenterol. 2014 Feb;49(2):222-37.

20 Pasha SF, Leighton JA, Das A, Harrison ME, Gurudu SR, Ramirez FC, et al. Comparison of the yield and miss rate of narrow band imaging and white light endoscopy in patients undergoing screening or surveillance colonoscopy: a meta-analysis. Am J Gastroenterol. 2012 Mar;107(3):363-70; quiz 371.

21 Horimatsu T, Sano Y, Tanaka S, Kawamura T, Saito S, Iwatate M, et al. Next-generation narrow band imaging system for colonic polyp detection: a prospective multicenter randomized trial. Int J Colorectal Dis. 2015 Jul;30(7): 947-54.

22 Leung WK, Lo OS, Liu KS, Tong T, But DY, Lam FY, et al. Detection of colorectal adenoma by narrow band imaging (HQ190) vs. high-definition white light colonoscopy: a randomized controlled trial. Am J Gastroenterol. 2014 Jun;109(6):855-63.

23 Ogiso K, Yoshida N, Siah KT, Kitae H, Murakami $\mathrm{T}$, Hirose $\mathrm{R}$, et al. New-generation narrow band imaging improves visibility of polyps: a colonoscopy video evaluation study. J Gastroenterol. 2016 Sep;51(9):883-90.

24 Bowman EA, Pfau PR, Mitra A, Reichelderfer M, Gopal DV, Hall BS, et al. High Definition Colonoscopy Combined with i-SCAN Imaging Technology Is Superior in the Detection of Adenomas and Advanced Lesions Compared to High Definition Colonoscopy Alone. Diagn Ther Endosc. 2015;2015:167406.

25 Hoffman A, Loth L, Rey JW, Rahman F, Goetz $\mathrm{M}$, Hansen T, et al. High definition plus colonoscopy combined with i-scan tone enhancement vs. high definition colonoscopy for colorectal neoplasia: A randomized trial. Dig Liver Dis. 2014 Nov;46(11):991-6.

26 Hoffman A, Kagel C, Goetz M, Tresch A, Mudter J, Biesterfeld S, et al. Recognition and characterization of small colonic neoplasia with high-definition colonoscopy using iScan is as precise as chromoendoscopy. Dig Liver Dis. 2010 Jan;42(1):45-50.

27 Testoni PA, Notaristefano C, Vailati C, Di Leo M, Viale E. High-definition colonoscopy with i-Scan: better diagnosis for small polyps and flat adenomas. World J Gastroenterol. 2012 Oct;18(37):5231-9.

28 Hoffman A, Sar F, Goetz M, Tresch A, Mudter J, Biesterfeld S, et al. High definition colonoscopy combined with i-Scan is superior in the detection of colorectal neoplasias compared with standard video colonoscopy: a prospective randomized controlled trial. Endoscopy. 2010 Oct;42(10):827-33.

29 Paggi S, Mogavero G, Amato A, Rondonotti E, Andrealli A, Imperiali G, et al. Linked color imaging reduces the miss rate of neoplastic lesions in the right colon: a randomized tandem colonoscopy study. Endoscopy. 2018 Apr; 50(4):396-402.

30 Oliveira Dos Santos CE, Malaman D, PereiraLima JC, de Quadros Onófrio F, Ribas Filho JM. Impact of linked-color imaging on colorectal adenoma detection. Gastrointest Endosc. 2019 Nov;90(5):826-34.

31 Fujimoto D, Muguruma N, Okamoto K, Fujino Y, Kagemoto K, Okada Y, et al. Linked color imaging enhances endoscopic detection of sessile serrated adenoma/polyps. Endosc Int Open. 2018 Mar;6(3):E322-34. 
32 Suzuki T, Hara T, Kitagawa Y, Takashiro H, Nankinzan R, Sugita O, et al. Linked-color imaging improves endoscopic visibility of colorectal nongranular flat lesions. Gastrointest Endosc. 2017 Oct;86(4):692-7.

33 Kessler WR, Imperiale TF, Klein RW, Wielage $\mathrm{RC}, \mathrm{Rex} \mathrm{DK}$. A quantitative assessment of the risks and cost savings of forgoing histologic examination of diminutive polyps. Endoscopy. 2011 Aug;43(8):683-91.

34 Kahi CJ, Anderson JC, Waxman I, Kessler WR, Imperiale TF, Li X, et al. High-definition chromocolonoscopy vs. high-definition white light colonoscopy for average-risk colorectal cancer screening. Am J Gastroenterol. 2010 Jun;105(6):1301-7.

35 Rex DK, Helbig CC. High yields of small and flat adenomas with high-definition colonoscopes using either white light or narrow band imaging. Gastroenterology. 2007 Jul;133(1): $42-7$

36 Gupta N, Bansal A, Rao D, Early DS, Jonnalagadda S, Wani SB, et al. Prevalence of advanced histological features in diminutive and small colon polyps. Gastrointest Endosc. 2012 May;75(5):1022-30.

37 O’Brien MJ, Winawer SJ, Zauber AG, Gottlieb LS, Sternberg SS, Diaz B, et al. The National Polyp Study. Patient and polyp characteristics associated with high-grade dysplasia in colorectal adenomas. Gastroenterology. 1990 Feb;98(2):371-9.

38 Butterly LF, Chase MP, Pohl H, Fiarman GS Prevalence of clinically important histology in small adenomas. Clin Gastroenterol Hepatol. 2006 Mar;4(3):343-8

39 Lieberman D, Moravec M, Holub J, Michaels L, Eisen G. Polyp size and advanced histology in patients undergoing colonoscopy screening: implications for CT colonography. Gastroenterology. 2008 Oct;135(4):1100-5

40 Rex DK, Kahi C, O’Brien M, Levin TR, Poh $\mathrm{H}$, Rastogi A, et al. The American Society for Gastrointestinal Endoscopy PIVI (Preservation and Incorporation of Valuable Endoscopic Innovations) on real-time endoscopic assessment of the histology of diminutive colorectal polyps. Gastrointest Endosc. 2011 Mar;73(3):419-22.

41 Hassan C, Pickhardt PJ, Rex DK. A resect and discard strategy would improve cost-effectiveness of colorectal cancer screening. Clin Gastroenterol Hepatol. 2010 Oct;8(10):8659, 9.e1-3

42 Hewett DG, Kaltenbach T, Sano Y, Tanaka S, Saunders BP, Ponchon T, et al. Validation of a simple classification system for endoscopic diagnosis of small colorectal polyps using narrow-band imaging. Gastroenterology. 2012 Sep;143(3):599-607.e1.

43 ASGE Technology Committee, Abu Dayyeh BK, Thosani N, Konda V, Wallace MB, Rex DK, et al. ASGE Technology Committee systematic review and meta-analysis assessing the ASGE PIVI thresholds for adopting realtime endoscopic assessment of the histology of diminutive colorectal polyps. Gastrointest Endosc. 2015 Mar;81(3):502.e1-16

44 Lieberman DA, Rex DK, Winawer SJ, Giardiello FM, Johnson DA, Levin TR. Guidelines for colonoscopy surveillance after screening and polypectomy: a consensus update by the
US Multi-Society Task Force on Colorectal Cancer. Gastroenterology. 2012 Sep;143(3): 844-57.

45 Basford PJ, Longcroft-Wheaton G, Higgins B, Bhandari P. High-definition endoscopy with i-Scan for evaluation of small colon polyps: the HiSCOPE study. Gastrointest Endosc. 2014 Jan;79(1):111-8

46 Klenske E, Zopf S, Neufert C, Nägel A, Siebler J, Gschossmann J, et al. I-scan optical enhancement for the in vivo prediction of diminutive colorectal polyp histology: results from a prospective three-phased multicentre trial. PLoS One. 2018 May;13(5):e0197520.

47 Rath T, Tontini GE, Nägel A, Vieth M, Zopf S, Günther C, et al. High-definition endoscopy with digital chromoendoscopy for histologic prediction of distal colorectal polyps. BMC Gastroenterol. 2015 Oct;15(1):145.

48 Longcroft-Wheaton G, Brown J, Cowlishaw D, Higgins B, Bhandari P. High-definition vs. standard-definition colonoscopy in the characterization of small colonic polyps: results from a randomized trial. Endoscopy. 2012 Oct;44(10):905-10.

49 Longcroft-Wheaton GR, Higgins B, Bhandari P. Flexible spectral imaging color enhancement and indigo carmine in neoplasia diagnosis during colonoscopy: a large prospective UK series. Eur J Gastroenterol Hepatol. 2011 Oct;23(10):903-11.

50 Kuiper T, Marsman WA, Jansen JM, van Soest EJ, Haan YC, Bakker GJ, et al. Accuracy for optical diagnosis of small colorectal polyps in nonacademic settings. Clin Gastroenterol Hepatol. 2012 Sep;10(9):1016-20; quiz e79.

51 Ladabaum U, Fioritto A, Mitani A, Desai M, Kim JP, Rex DK, et al. Real-time optical biopsy of colon polyps with narrow band imaging in community practice does not yet meet key thresholds for clinical decisions. Gastroenterology. 2013 Jan;144(1):81-91.

52 Rastogi A, Rao DS, Gupta N, Grisolano SW, Buckles DC, Sidorenko E, et al. Impact of a computer-based teaching module on characterization of diminutive colon polyps by using narrow-band imaging by non-experts in academic and community practice: a videobased study. Gastrointest Endosc. 2014 Mar; 79(3):390-8

53 Vu HT, Sayuk GS, Hollander TG, Clebanoff J, Edmundowicz SA, Gyawali CP, et al. Resect and discard approach to colon polyps: realworld applicability among academic and community gastroenterologists. Dig Dis Sci. 2015 Feb;60(2):502-8.

54 Rex DK, Patel NJ, Vemulapalli KC. A survey of patient acceptance of resect and discard for diminutive polyps. Gastrointest Endosc. 2015 Aug;82(2):376-80.e1.

55 Sakata S, Lee AHS, Kheir AO, Tutticci NJ, Naidu S, Stevenson ARL, et al. Patient acceptance of the optical diagnosis and misdiagnosis of diminutive colorectal polyps. Gastrointest Endosc. 2017 Aug;86(2):372-5.e2.

56 Butterly L, Robinson CM, Anderson JC, Weiss JE, Goodrich M, Onega TL, et al. Serrated and adenomatous polyp detection increases with longer withdrawal time: results from the New Hampshire Colonoscopy Registry. Am J Gastroenterol. 2014 Mar;109(3): 417-26.
57 de Wijkerslooth TR, Stoop EM, Bossuyt PM, Tytgat KM, Dees J, Mathus-Vliegen EM, et al. Differences in proximal serrated polyp detection among endoscopists are associated with variability in withdrawal time. Gastrointest Endosc. 2013 Apr;77(4):617-23.

58 IJspeert JE, Bevan R, Senore C, Kaminski MF, Kuipers EJ, Mroz A, et al. Detection rate of serrated polyps and serrated polyposis syndrome in colorectal cancer screening cohorts: a European overview. Gut. 2017 Jul;66(7): 1225-32.

59 Kahi CJ, Hewett DG, Norton DL, Eckert GJ, Rex DK. Prevalence and variable detection of proximal colon serrated polyps during screening colonoscopy. Clin Gastroenterol Hepatol. 2011 Jan;9(1):42-6.

60 Vleugels JL, IJspeert JE, Hazewinkel Y, van der Vlugt M, Fockens P, Koens L, et al. Optical Diagnosis of Sessile Serrated Polyps: Bottleneck for the Optical Diagnosis Paradigm? J Clin Gastroenterol. 2017 May/Jun;51(5):426-32.

61 Kiesslich R, Burg J, Vieth M, Gnaendiger J, Enders M, Delaney P, et al. Confocal laser endoscopy for diagnosing intraepithelial neoplasias and colorectal cancer in vivo. Gastroenterology. 2004 Sep;127(3):706-13.

62 Sanduleanu S, Driessen A, Gomez-Garcia E, Hameeteman W, de Bruïne A, Masclee A. In vivo diagnosis and classification of colorectal neoplasia by chromoendoscopy-guided confocal laser endomicroscopy. Clin Gastroenterol Hepatol. 2010 Apr;8(4):371-8.

63 Buchner AM, Shahid MW, Heckman MG, Krishna M, Ghabril M, Hasan M, et al. Comparison of probe-based confocal laser endomicroscopy with virtual chromoendoscopy for classification of colon polyps. Gastroenterology. 2010 Mar;138(3):834-42.

64 André B, Vercauteren T, Buchner AM, Krishna M, Ayache N, Wallace MB. Software for automated classification of probe-based confocal laser endomicroscopy videos of colorectal polyps. World J Gastroenterol. 2012 Oct; 18(39):5560-9.

65 Buchner AM, Gomez V, Heckman MG, Shahid MW, Achem S, Gill KR, et al. The learning curve of in vivo probe-based confocal laser endomicroscopy for prediction of colorectal neoplasia. Gastrointest Endosc. 2011 Mar; 73(3):556-60

66 Gómez V, Buchner AM, Dekker E, van den Broek FJ, Meining A, Shahid MW, et al. Interobserver agreement and accuracy among international experts with probe-based confocal laser endomicroscopy in predicting colorectal neoplasia. Endoscopy. 2010 Apr; 42(4):286-91

67 Kuiper T, van den Broek FJ, van Eeden S, Fockens P, Dekker E. Feasibility and accuracy of confocal endomicroscopy in comparison with narrow-band imaging and chromoendoscopy for the differentiation of colorectal lesions. Am J Gastroenterol. 2012 Apr;107(4):543-50.

68 De Palma GD, Staibano S, Siciliano S, Persico $\mathrm{M}$, Masone S, Maione F, et al. In vivo characterisation of superficial colorectal neoplastic lesions with high-resolution probe-based confocal laser endomicroscopy in combination with video-mosaicing: a feasibility study to enhance routine endoscopy. Dig Liver Dis. 2010 Nov;42(11):791-7. 
69 Shahid MW, Buchner AM, Raimondo M, Woodward TA, Krishna M, Wallace MB. Accuracy of real-time vs. blinded offline diagnosis of neoplastic colorectal polyps using probe-based confocal laser endomicroscopy: a pilot study. Endoscopy. 2012 Apr;44(4): 343-8.

70 Kim B, Kim YH, Park SJ, Cheon JH, Kim TI, Kim WH, et al. Probe-based confocal laser endomicroscopy for evaluating the submucosal invasion of colorectal neoplasms. Surg Endosc. 2017 Feb;31(2):594-601.

71 Kudo SE, Wakamura K, Ikehara N, Mori Y, Inoue $\mathrm{H}$, Hamatani S. Diagnosis of colorectal lesions with a novel endocytoscopic classification - a pilot study. Endoscopy. 2011 Oct; 43(10):869-75.

72 Kudo SE, Misawa M, Wada Y, Nakamura H Kataoka S, Maeda Y, et al. Endocytoscopic microvasculature evaluation is a reliable new diagnostic method for colorectal lesions (with video). Gastrointest Endosc. 2015 Nov;82(5): 912-23.

73 Kudo T, Kudo SE, Wakamura K, Mori Y, Misawa M, Hayashi T, et al. Diagnostic performance of endocytoscopy for evaluating the invasion depth of different morphological types of colorectal tumors. Dig Endosc. 2015 Nov;27(7):754-61

74 Kutsukawa M, Kudo SE, Ikehara N, Ogawa Y, Wakamura K, Mori Y, et al. Efficiency of endocytoscopy in differentiating types of serrated polyps. Gastrointest Endosc. 2014 Apr; 79(4):648-56.

75 Ogawa Y, Kudo SE, Mori Y, Ikehara N, Maeda Y, Wakamura K, et al. Use of endocytoscopy for identification of sessile serrated adenoma/ polyps and hyperplastic polyps by quantitative image analysis of the luminal areas. Endosc Int Open. 2017 Aug;5(8):E769-74.

76 Kudo SE, Misawa M, Mori Y, Hotta K, Ohtsuka $\mathrm{K}$, Ikematsu $\mathrm{H}$, et al. Artificial Intel- ligence-assisted System Improves Endoscopic Identification of Colorectal Neoplasms. Clin Gastroenterol Hepatol. 2019 Sep;S15423565(19)30997-8.

77 Misawa M, Kudo SE, Mori Y, Takeda K, Maeda Y, Kataoka S, et al. Accuracy of computeraided diagnosis based on narrow-band imaging endocytoscopy for diagnosing colorectal lesions: comparison with experts. Int J CARS 2017 May;12(5):757-66.

78 Mori Y, Kudo SE, Chiu PW, Singh R, Misawa M, Wakamura K, et al. Impact of an automated system for endocytoscopic diagnosis of small colorectal lesions: an international webbased study. Endoscopy. 2016 Dec;48(12): 1110-8.

79 Mori Y, Kudo SE, Wakamura K, Misawa M, Ogawa Y, Kutsukawa M, et al. Novel computer-aided diagnostic system for colorectal lesions by using endocytoscopy (with videos). Gastrointest Endosc. 2015 Mar;81(3):621-9. 